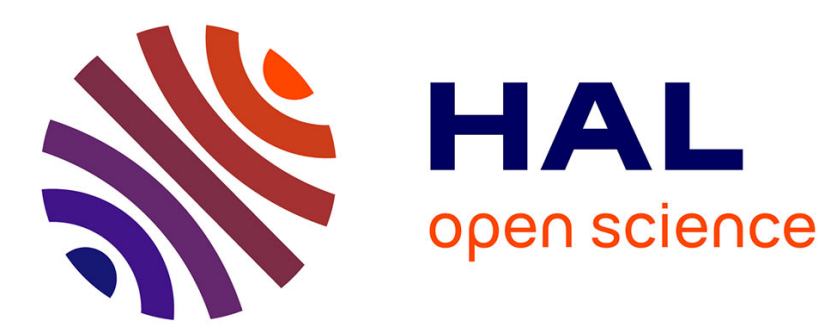

\title{
Guaranteed State and Parameter Estimation for Nonlinear Dynamical Aerospace Models
}

Qiaochu Li, Carine Jauberthie, Lilianne Denis-Vidal, Zohra Cherfi

\section{To cite this version:}

Qiaochu Li, Carine Jauberthie, Lilianne Denis-Vidal, Zohra Cherfi. Guaranteed State and Parameter Estimation for Nonlinear Dynamical Aerospace Models. Informatics in Control, Automation and Robotics (ICINCO), Sep 2014, Vienna, Austria. pp.510-527, 10.5220/0005053105190527 . hal01136619

\section{HAL Id: hal-01136619 \\ https://hal.science/hal-01136619}

Submitted on 27 Mar 2015

HAL is a multi-disciplinary open access archive for the deposit and dissemination of scientific research documents, whether they are published or not. The documents may come from teaching and research institutions in France or abroad, or from public or private research centers.
L'archive ouverte pluridisciplinaire HAL, est destinée au dépôt et à la diffusion de documents scientifiques de niveau recherche, publiés ou non, émanant des établissements d'enseignement et de recherche français ou étrangers, des laboratoires publics ou privés. 


\title{
Guaranteed state and parameter estimation for nonlinear dynamical aerospace models
}

\author{
Qiaochu $\mathrm{Li}^{1}$, Carine Jauberthie ${ }^{2,3}$, Lilianne Denis-Vidal ${ }^{1}$ and Zohra Cherfi ${ }^{1}$ \\ ${ }^{1}$ University of Technology of Compiègne, Compiègne, France \\ ${ }^{2}$ CNRS, LAAS, 7 avenue du Colonel Roche, F-31400 Toulouse, France, \\ ${ }^{3}$ Univ de Toulouse, UPS, LAAS, F-31400 Toulouse, France \\ \{qiaochu.li, zohra.cherfi\}@utc.fr, cjaubert@laas.fr, lilianne.denis-vidal@math.univ-lille1.fr
}

\begin{abstract}
Keywords: Parameter estimation; State estimation; Continuous-time systems; Nonlinear systems; Bounded noise; Interval analysis; Aerospace models.

Abstract: This paper deals with parameter and state estimation in a bounded-error context for uncertain dynamical aerospace models when the input is considered optimized or not. In a bounded-error context, perturbations are assumed bounded but otherwise unknown. The parameters to be estimated are also considered bounded. The tools of the presented work are based on a guaranteed numerical set integration solver of ordinary differential equations combined with adapted set inversion computation. The main contribution of this work consists in developing procedures for parameter estimation whose performance is highly related with the input of system. In this paper, a comparison with a classical non-optimized input is proposed.
\end{abstract}

\section{INTRODUCTION}

Complex systems are often subjected to uncertainties that make the modeling task awkward. These uncertainties can be unstructured when the equations of the system are not entirely known or structured when the equations are known but not the values of their parameters. In both cases, it is particularly difficult to get an accurate model of the perturbations and noises acting on the system. This may turn the usual stochastic framework inappropriate. Thus, we prefer to deal with set-membership framework in which perturbations and noises are assumed to be bounded but otherwise unknown. In this framework, we obtain "guaranteed solutions". This last expression means that for all conditions belonging to a bounded set, the obtained set contains all the solutions.

Guaranteed state and parameter estimation methods are an interesting alternative to stochastic model based estimation when perturbations and noises are assumed to be bounded but otherwise unknown. These methods have received a lot of attention in the last years and the literature on this topic shows interesting progress, for example (Kieffer et al., 2002), (Deville et al., 2002), (Jaulin, 2009), (Rauh and Auer, 2011), (Pasca, 2010) or for example (Jauberthie et al., 2013).

Moreover, experimental design is important for identifying mathematical models of modern aircraft dynamics from flight test data, for example. In the case of aerospace domain, the flight test input has a major impact on the quality of the data for modeling purposes. Good experimental design must account for practical constraints during the test. The overall goal is to design an experiment that produces data from which model parameters can be estimated accurately. Most importantly, in an estimation framework, the experimental conditions about noise and disturbances are usually properly modeled through appropriate assumptions about probability distributions ((Mehra, 1974), (Walter and Pronzato, 1994), (Kiefer, 1974)). The conventional approach for the experimental design is based on stochastic models for uncertain parameters and measurement errors (see for example (Rojas et al., 2007)). However, other sources of uncertainty are not well-suited to the stochastic approach and are better modeled as bounded uncertainty. This is the case of parameter uncertainties that generally arise from design tolerances and from aging. In such cases, combining stochastic and bounded uncertainties may be an appropriate solution. Some works consider that the parameters belong to some prior domain, on which no probability function has to be defined (for example (Pronzato and Walter, 1988), (Belforte and Gay, 2004)). The first aim at optimizing is the worst possible performance of the experi- 
ment over the prior domain for the parameters. This maximin approach to synthesis the optimal input is described and the specific criterion are developped.

In a recent paper (Jauberthie and Chanthery, 2013), it is supposed that the uncertainty on parameters can be modelled by bounded intervals and the concepts of interval analysis are used for the optimal input synthesis. In this paper the original approach of optimal input design for uncertain bounded parameter estimation is an extension of the works of E.A. Morelli ((Morelli, 1999)) using the dynamic programming. This approach combines the concepts of dynamical programming with the maximin approach and with the tools of interval analysis. In the presented work, we propose to apply an optimal input obtained in (Jauberthie and Chanthery, 2013) for the same case study taken from aerospace domain. By using this optimal input, we obtain an original algorithm to achieve a guaranteed state and parameter estimation based on interval analysis.

This paper is organized as follows. In Section 2, the problem statement and the case study are presented. The case study is taken from aerospace domain and describes the longitudinal motion of a glider. Section 3 presents some basic tools of interval analysis. The notions of interval, box, interval matrix and inclusion function are given. Section 4 presents the fundamental algorithm to implement state and parameter estimation. In Section 5, the estimation results obtained on the case study are presented and discussed. Two cases of inputs are tested and the performance of the optimal input is highlighted. Finally, some conclusions are outlined in section 6 .

\section{PROBLEM FORMULATION AND CASE STUDY}

This paper deals with estimating the unknown state and parameters for a nonlinear dynamic system of the following form:

$$
\left\{\begin{array}{l}
\dot{x}(t, p)=f(x(t, p), p)+u(t) g(x(t, p), p)), \\
y(t, p)=h(x(t, p), p), \quad x(0) \in\left[\mathrm{X}_{0}\right], p \in\left[P_{0}\right],
\end{array}\right.
$$

where $x(t, p) \in \mathbb{R}^{n}$ and $y(t, p) \in \mathbb{R}^{n_{y}}$ denote respectively the state variables and the measured outputs. The initial conditions $x(0)$ are supposed to belong to an initial bounded box $\left[\mathrm{X}_{0}\right] . u(t)$ represents the input.

The vector $p$ is the vector of parameters to be estimated and $p \in \mathbb{R}^{n_{p}}$ which is supposed to belong to an a priori box $\left[P_{0}\right]$.

The time $t$ is assumed to belong to $\left[0, t_{\max }\right]$. The functions $f, g$ and $h$ are nonlinear functions. $f$ and $g$ are supposed analytic on $M$ for every $p \in\left[p_{0}\right]$, where $M$ is an open set of $\mathbb{R}^{n}$ such that $x(t, p) \in M$ for every $p \in\left[p_{0}\right]$ and $\left.t \in\left[0, t_{\max }\right]\right)$.

The output error is assumed to be given by:

$$
v\left(t_{i}\right)=y_{m}\left(t_{i}\right)-y\left(t_{i}, p\right), \quad i=1, \ldots, N .
$$

We assume that $\underline{v}\left(t_{i}\right)$ and $\bar{v}\left(t_{i}\right)$ are known as lower and upper bounds for the acceptable output errors. Such bounds may, for instance, correspond to a bounded measurement noise. The integer $N$ is the total number of sample times.

Interval arithmetic is used to compute guaranteed bounds for the considered problem at the sampling times $\left\{t_{1}, t_{2}, \ldots, t_{N}\right\}$.

The case study that we consider in this work is given by an aerospace model which describes the longitudinal motion of a glider. The projection of the general equations of motion onto the aerodynamic reference frame of the aircraft and the linearization of aerodynamic coefficients give the following system:

$$
\left\{\begin{aligned}
\dot{V}= & -g \sin (\theta-\alpha)-\frac{1}{2 m} \rho S V^{2}\left(C_{x}^{0}\right. \\
& \left.+C_{x \alpha}\left(\alpha-\alpha_{0}\right)+C_{x \delta_{m}}\left(\delta_{m}-\delta_{m_{0}}\right)\right), \\
\dot{\alpha}= & \frac{2}{2 m V+\rho S l V C_{z \dot{\alpha}}}\left\{m V q+m g \frac{\cos (\theta-\alpha)}{V}\right. \\
& -\frac{1}{2} \rho S V^{2}\left(C_{z}^{0}+C_{z \alpha}\left(\alpha-\alpha_{0}\right)\right. \\
& \left.\left.+C_{z q} \frac{q l}{V}+C_{z \delta_{m}}\left(\delta_{m}-\delta_{m_{0}}\right)\right)\right\}, \\
\dot{q}= & \frac{1}{2 B} \rho S l V^{2}\left\{C_{m}^{0}+C_{m \alpha}\left(\alpha-\alpha_{0}\right)+C_{m q} \frac{q l}{V}\right. \\
& +C_{m \dot{\alpha}} \frac{2 l}{2 m V^{2}+\rho S l V^{2} C_{z \dot{\alpha}}}[m V q \\
& +m g \frac{\cos (\theta-\alpha)}{V} \\
& -\frac{1}{2} \rho S V^{2}\left(C_{z}^{0}+C_{z \alpha}\left(\alpha-\alpha_{0}\right)+C_{z q} \frac{q l}{V}\right. \\
& \left.\left.\left.+C_{z \delta_{m}}\left(\delta_{m}-\delta_{m_{0}}\right)\right)\right]+C_{m \delta_{m}}\left(\delta_{m}-\delta_{m_{0}}\right)\right\}, \\
\dot{\theta}= & q .
\end{aligned}\right.
$$

In these equations, the state vector $x$ is given by $(V, \alpha, q, \theta)^{\top}$, the observation $y$ is full (i.e., $\left.y=x\right)$, the input $u$ is $\delta_{m}\left(\delta_{m_{0}}\right.$ represents the initial condition). The variable $V$ denotes the speed of the aircraft, $\alpha$ the angle of attack, $\alpha_{0}$ the trim value of $\alpha, \theta$ the pitch angle, $q$ the pitch rate, $\delta_{m}$ the elevator deflection angle, $\rho$ the air density, $g$ the acceleration due to gravity, $l$ a reference length and $S$ the area of a reference surface. $B$ represents a moment of inertia. The parameters to be estimated are $C_{z \dot{\alpha}}, C_{z q}, C_{m \dot{\alpha}}, C_{m q}$, which are assumed to be uncertain. The other coefficients correspond to the dynamic stability derivatives are supposed to be known. 


\section{BASIC TOOLS}

Interval analysis provides tools for computing with sets which are described using outerapproximations formed by union of non-overlapping boxes. The following results are mainly taken from (Jaulin et al., 2001).

\subsection{Basic tools}

A real interval $[u]=[\underline{u}, \bar{u}]$ is a closed and connected subset of $\mathbb{R}$ where $\underline{u}$ represents the lower bound of $[u]$ and $\bar{u}$ represents the upper bound. The width of an interval $[u]$ is defined by $w([u])=\bar{u}-\underline{u}$, and its midpoint by $m([u])=(\bar{u}+\underline{u}) / 2$.

The set of all real intervals of $\mathbb{R}$ is denoted $\mathbb{I}$.

Two intervals $[u]$ and $[v]$ are equal if and only if $\underline{u}=\underline{v}$ and $\bar{u}=\bar{v}$. Real arithmetic operations are extended to intervals (Moore, 1966).

Arithmetic operations on two intervals $[u]$ and $[v]$ can be defined by:

$\circ \in\{+,-, *, /\},[u] \circ[v]=\{x \circ y \mid x \in[u], y \in[v]\}$.

An interval vector (or box) $[X]$ is a vector with interval components and may equivalently be seen as a cartesian product of scalar intervals:

$$
[X]=\left[x_{1}\right] \times\left[x_{2}\right] \ldots \times\left[x_{n}\right] .
$$

The set of $n$-dimensional real interval vectors is denoted by $\mathbb{I} \mathbb{R}^{n}$.

An interval matrix is a matrix with interval components. The set of $n \times m$ real interval matrices is denoted by $\mathbb{I} \mathbb{R}^{n \times m}$. The width $w($.$) of an interval vec-$ tor (or of an interval matrix) is the maximum of the widths of its interval components. The midpoint $m($. of an interval vector (resp. an interval matrix) is a vector (resp. a matrix) composed of the midpoint of its interval components.

Classical operations for interval vectors (resp. interval matrices) are direct extensions of the same operations for punctual vectors (resp. punctual matrices) (Moore, 1966).

Let $f: \mathbb{R}^{n} \rightarrow \mathbb{R}^{m}$, the range of the function $f$ over an interval vector $[u]$ is given by:

$$
f([u])=\{f(x) \mid x \in[u]\} .
$$

The interval function denoted $[f]$ is a function from $\mathbb{I R}^{n}$ to $\mathbb{R}^{m}$. It is an inclusion function for $f$ if:

$$
\forall[u] \in \mathbb{I}^{n}, \quad f([u]) \subseteq[f]([u]) .
$$

An inclusion function of $f$ can be obtained by replacing each occurrence of a real variable by its corresponding interval and by replacing each standard function by its interval evaluation. Such a function is called the natural inclusion function. In practice the inclusion function is not unique, it depends on the syntax of $f$.

\subsection{Set Inversion}

Consider the problem of determining a solution set for the unknown quantities $u$ defined by:

$$
\mathrm{S}=\{u \in \mathrm{U} \mid \Phi(u) \in[y]\}=\Phi^{-1}([y]) \cap \mathrm{U},
$$

where $[y]$ is known a priori, $\mathrm{U}$ is an a priori search set for $u$ and $\Phi$ a nonlinear function not necessarily invertible in the classical sense. (4) involves computing the reciprocal image of $\Phi$ and is known as a set inversion problem which can be solved using the algorithm Set Inverter Via Interval Analysis (denoted SIVIA). The algorithm SIVIA proposed in (Jaulin and Walter, 1993) is a recursive algorithm which explores all the search space without losing any solution. This algorithm makes it possible to derive a guaranteed enclosure of the solution set $\mathrm{S}$ as follows:

$$
\underline{\mathrm{S}} \subseteq \mathrm{S} \subseteq \overline{\mathrm{S}}
$$

The inner enclosure $\underline{S}$ is composed of the boxes that have been proved feasible. To prove that a box $[u]$ is feasible it is sufficient to prove that $\Phi([u]) \subseteq[y]$. Reversely, if it can be proved that $\Phi([u]) \cap[y]=\emptyset$, then the box $[u]$ is unfeasible. Otherwise, no conclusion can be reached and the box $[u]$ is said undetermined. The latter is then bisected and tested again until its size reaches a user-specified precision threshold $\varepsilon>0$. Such a termination criterion ensures that SIVIA terminates after a finite number of iterations.

\section{GUARANTEED STATE AND PARAMETER ESTIMATION}

This section concerns the integration of (1) and set inversion computation. Thus, the objective of this section is fist to obtain the state vector $x$ at the sampling times $\left\{t_{1}, t_{2}, \ldots, t_{N}\right\}$ corresponding to the measurement times of the outputs. Second follow the SIVIA procedure to get the validated sets of feasible parameters.

We note $\left[x_{j}\right]$ the box $\left[x\left(t_{j}\right)\right]$ where $t_{j}$ represents the sampling time, $j=1, \ldots, N$ and $x_{j}$ represents the solution of (1) at $t_{j}$. 


\subsection{Validated Integration for Nonlinear Systems}

Rigorous solution for dynamical nonlinear systems can be solved efficiently by considering methods based on Taylor expansions (Moore, 1966), (Rihm, 1994), (Berz and Makino, 1998) or (Nedialkov and Jackson, 2001). These methods consist in two parts: the first one verifies the existence and uniqueness of the solution by using the fixed point theorem and the Picard-Lindelöf operator. At a time $t_{j+1}$, an a priori box $\left[\tilde{x}_{j}\right]$ containing all solutions corresponding to all possible trajectories between $t_{j}$ and $t_{j+1}$ is computed. In the second part, the solution at $t_{j+1}$ is computed by using a Taylor expansion, where the remainder term is $\left[\tilde{x}_{j}\right]$.

To obtain the set $\left[\tilde{x}_{j}\right]$, a classical technique consists in inflating this set until it verifies the following inclusion (Lohner, 1987), (Nedialkov and Jackson, 2001):

$$
\left[x_{j}\right]+h_{j} f\left(\left[\tilde{x}_{j}\right]\right) \subseteq\left[\tilde{x}_{j}\right],
$$

where $h_{j}$ denotes the integration step and $\left[x_{j}\right]$ the first solution. In the proposed work, to state estimate, we use the package VNODE in which the previous validated integration method is implemented. The package VNODE, developed by N.S Nedialkov (Nedialkov et al., 2001), is a $C^{++}$package for computing bounds of solutions in Initial Value Problem for ordinary differential equation. In the latest version, named VNODE-LP, algorithms corresponding to high order enclosure and Hermite- Obreschkoff method (Nedialkov, 2006) have been implemented. Thus VNODE-LP gives a way to obtain tighter enclosure.

\subsection{Parameter Estimation}

Parameters estimation from experimental measures are usually obtained within a stochastic framework in which known distribution laws are associated to interferences and measurement noise. Oppositely, in a bounded error context, measures and modeling errors are supposed to be unknown but to stay within known and acceptable bounds.

Errors between measured and predicted outputs may rely on many factors, among them: limited sensors accuracy, interferences, noise, structured uncertainties, etc. Some are quantifiable, some are not. We consider here the quantifiable error $v$, which is added to the model output $y$. The experimental outputs $y_{m}$ are given by (Equation (2)):

$$
y_{m}\left(t_{i}\right)=y\left(t_{i}, p\right)+\mathrm{v}\left(t_{i}\right), 1 \leq i \leq N .
$$

In the presented work, the error $v$ is supposed to be within an interval whose lower bound is $\underline{v}$ and upper bound is $\bar{v}$. An allowable error set $\mathbb{E}$ may be defined as a set of constraints:

$$
\mathbb{E}=\left\{v\left(t_{i}\right) \mid \underline{v}\left(t_{i}\right) \leq v\left(t_{i}\right) \leq \bar{v}\left(t_{i}\right)\right\} .
$$

These bounds may be considered constant over time as well as variable. They may be established from data given by constructors for electronic parts for example.

A parameter vector $p$ is acceptable if and only if the error between $y_{m}$ and the model output $y$ is bounded in a known way. To estimate system parameters, we have to get the set $\mathbb{P}$ of all parameters $p$ enclosed in the a priori search set $\left[P_{0}\right]$ such that error between real data and model outputs belongs to $\mathbb{E}$ :

$$
\begin{aligned}
\mathbb{P} & =\left\{p \in\left[P_{0}\right] \mid y_{m}\left(t_{i}\right)-y\left(t_{i}, p\right) \in\left[\underline{v}_{i}, \bar{v}_{i}\right], \forall i=1, \cdots, N\right\}, \\
& =\left\{p \in\left[P_{0}\right] \mid\left[\mathrm{v}\left(t_{i}\right)\right] \in \mathbb{E}, \forall i=1, \cdots, N\right\} .
\end{aligned}
$$

The characterization of the set $\mathbb{P}$ may be defined as a set inversion problem (4). By simplicity of notation, we note this set:

$$
\mathbb{P}=\left[\mathrm{v}^{-1}\right](\mathbb{E}) \cap\left[P_{0}\right] .
$$

A guaranteed enclosure of $\mathbb{P}$ may be computed by using the SIVIA algorithm presented in Section 3.2.

\subsection{Parameter and state estimation}

To perform the state and parameter estimation, we propose the following algorithm. This algorithm has been implemented in $\mathrm{C}++$. It combines the strategy of bisections used in SIVIA and the validated integration used by VNODE. A threshold $\varepsilon$ is considered for the bisections in SIVIA. The choice of this threshold depends on the a priori initial box of parameters to be estimated. In this algorithm, the function bisectBox divides a box into two sub-boxes and the function VNODELP is the call to the software VNODE-LP. 


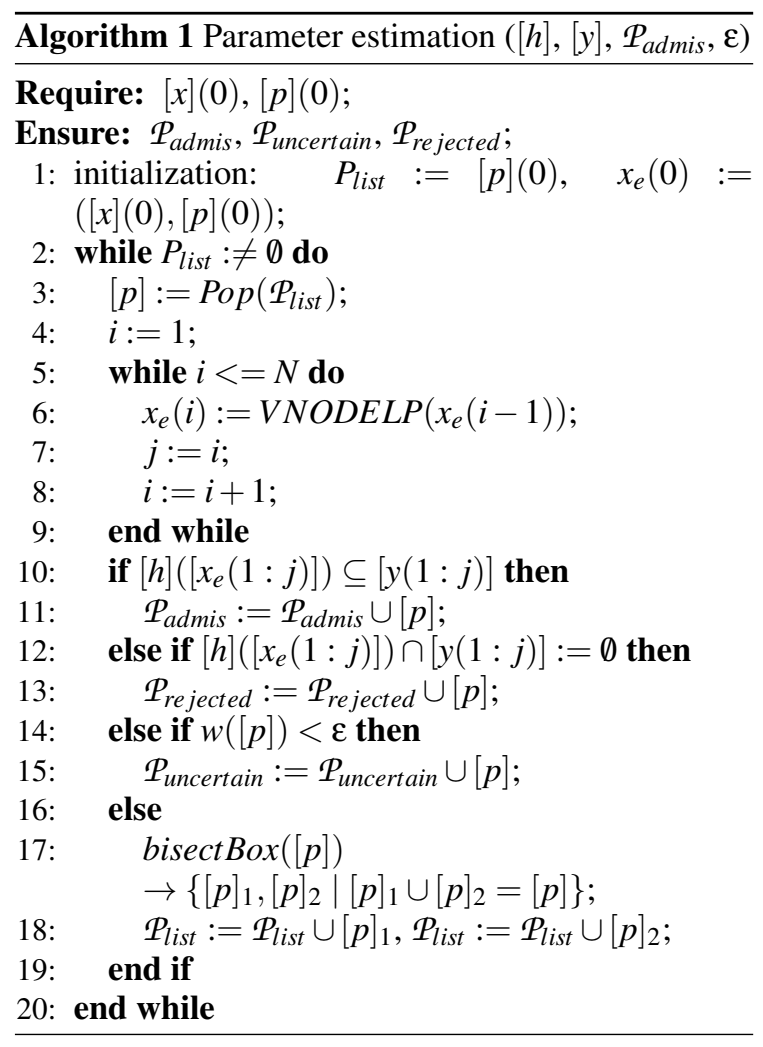

\section{APPLICATION}

In this section, the state and parameter estimation of the aerospace system is performed by using the proposed algorithm. The initial conditions are supposed to belong to:

$$
\left[X_{0}\right]=\left[\begin{array}{cc}
28.48 & 28.52 \\
6.2682 & 6.7265 \\
-0.2292 & 0.2292 \\
2.2002 & 2.6585
\end{array}\right]
$$

The parameters are supposed to be included in:

$$
\left[P_{0}\right]=\left[\begin{array}{cc}
1.71 & 1.89 \\
4.75 & 5.25 \\
-5.25 & -4.75 \\
-23.1 & -20.9
\end{array}\right]
$$

The output error (2) is supposed to be bounded by:

$$
[v]=\left[\begin{array}{ll}
-0.0447 & 0.0447 \\
-0.0044 & 0.0044 \\
-0.0044 & 0.0044 \\
-0.0044 & 0.0044
\end{array}\right]
$$

The measurements have been simulated by using the parameters equal to $(1.8,5,-5,-22)$ and initial states $\left[\mathrm{X}_{0}\right]$. The test duration is fixed at one second. The stop criterion for SIVIA is $\varepsilon=[0.01,0.05,0.05,0.1]$ that means that the stop threshold for the first parameter is 0.01 , the second and third are 0.05 and the last one is 0.1 .

Two cases of inputs are considered for the tests: the first one concerns a constant input and the second one is an optimal input proposed in (Jauberthie and Chanthery, 2013) with six stages. The optimal input is the following:

$$
\begin{aligned}
u(t) & =\delta_{m 0}+a_{6_{0}} H\left(t-t_{0_{6}}\right)-2 a_{6_{1}} H\left(t-t_{1_{6}}\right) \\
& +2 a_{6_{2}} H\left(t-t_{2_{6}}\right)-2 a_{6_{3}} H\left(t-t_{3_{6}}\right)+ \\
& 2 a_{6_{4}} H\left(t-t_{4_{6}}\right)-2 a_{6_{5}} H\left(t-t_{5_{6}}\right)
\end{aligned}
$$

with $a_{6_{i}}=1.6$ degrees with $i=0, \cdots, 5$ and , $t_{0_{6}}=$ $0 s, a_{1_{6}}=0.1667 s, a_{2_{6}}=0.3334 s, a_{3_{6}}=0.5001 s$, $a_{4_{6}}=0.6668 s a_{56}=0.8335 s$. The function $H$ is the Heaviside function.

The optimized input is given in the Figure 1:

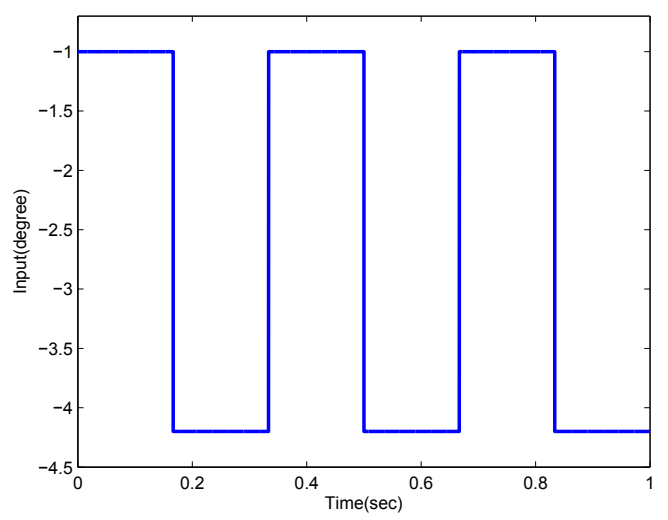

Figure 1: Optimized input for six stages.

The order of the Taylor expansion is chosen automatically by the VNODE-LP.

The parameter estimation results, for a constant input, are given in Figures 2, 3, 4, 5, 6 and 7. In these figures, the red boxes represent the acceptable sets for parameters, the blue boxes represent the rejected boxes and the yellow boxes represent the undetermined boxes. The black border cube represent the box $\left[P_{0}\right]$. 


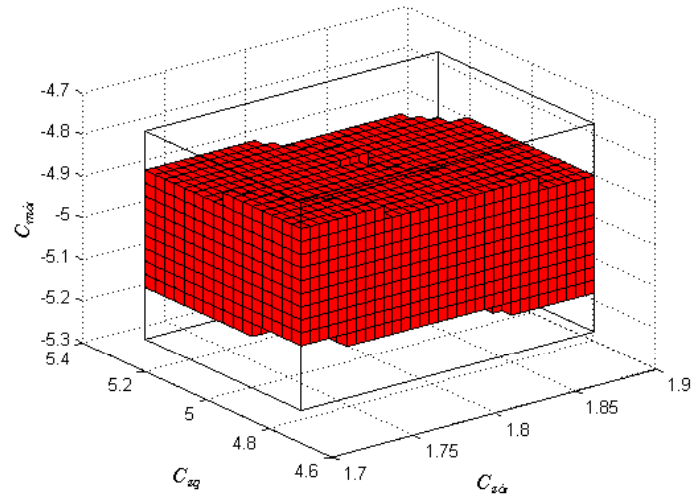

Figure 2: Acceptable sets $C_{z \dot{\alpha}}, C_{z q}, C_{m \dot{\alpha}}$ with constant input.

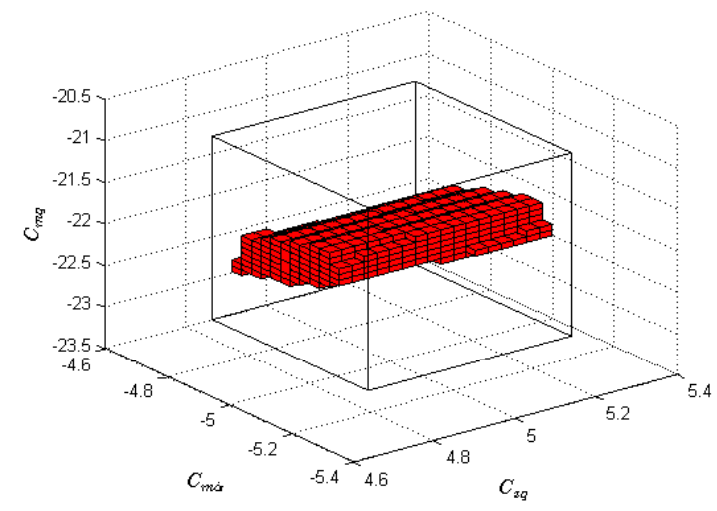

Figure 3: Acceptable sets $C_{z q}, C_{m \dot{\alpha}}, C_{m q}$ with constant input.

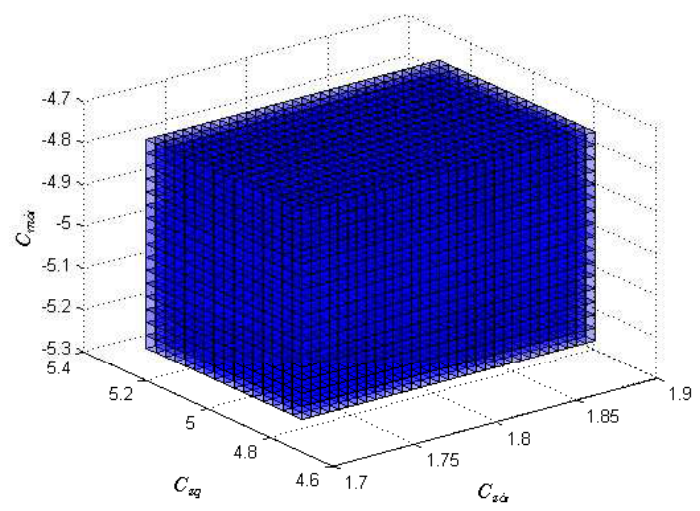

Figure 4: Rejected sets $C_{z \dot{\alpha}}, C_{z q}, C_{m \dot{\alpha}}$ with constant input.

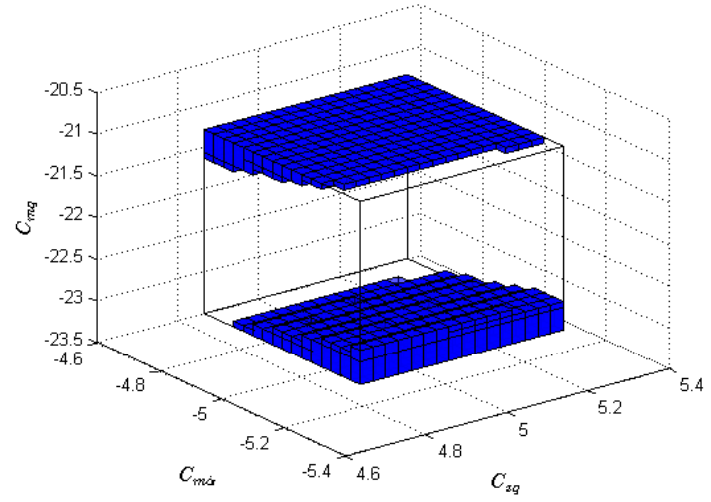

Figure 5: Rejected sets $C_{z q}, C_{m \dot{\alpha}}, C_{m q}$, with constant input.

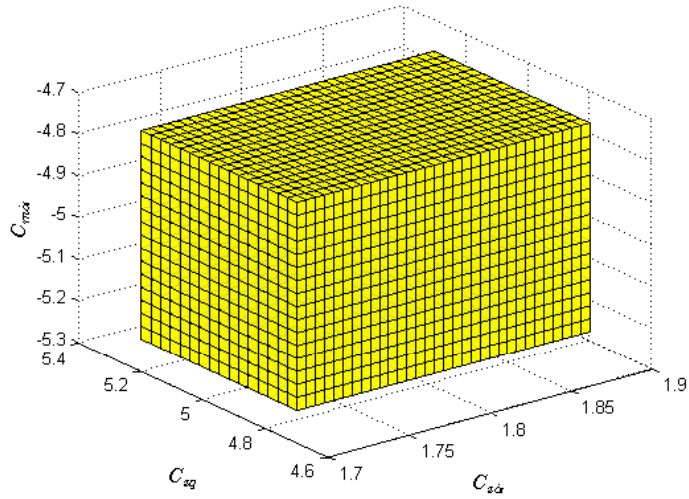

Figure 6: Undetermined sets $C_{z \dot{\alpha}}, C_{z q}$ and $C_{m \dot{\alpha}}$ with constant input.

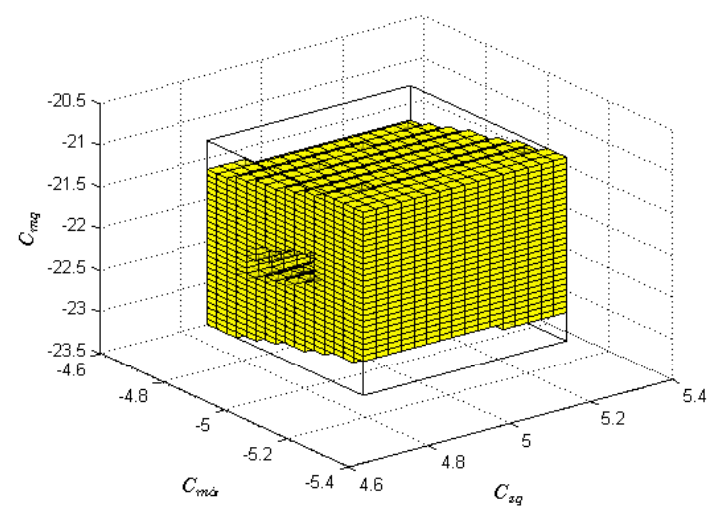

Figure 7: Undetermined sets $C_{z q}, C_{m \dot{\alpha}}, C_{m q}$, with constant input.

As seen in these figures, the first three parameters have not been well estimated. The diameter of each interval remained almost as proposed. The parameter $C_{m q}$ has been well obtained compared with other 
parameters.

By using the optimal input, we obtain Figures 8, $9,10,11,12$ and 13 .

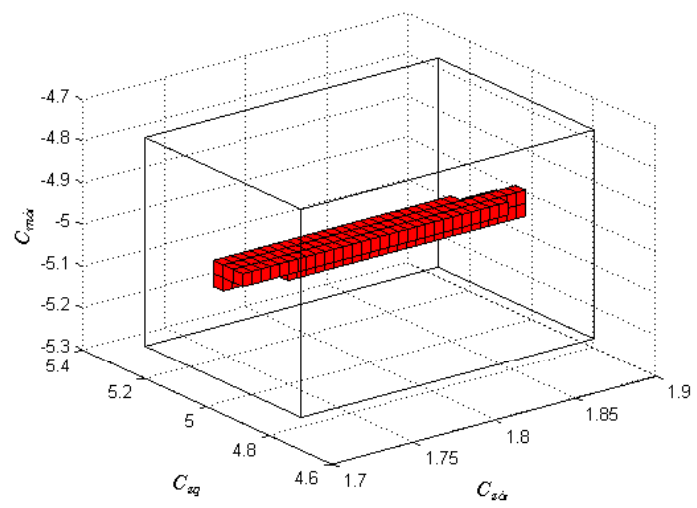

Figure 8: Acceptable sets $C_{z \dot{\alpha}}, C_{z q}, C_{m \dot{\alpha}}$ with optimal input.

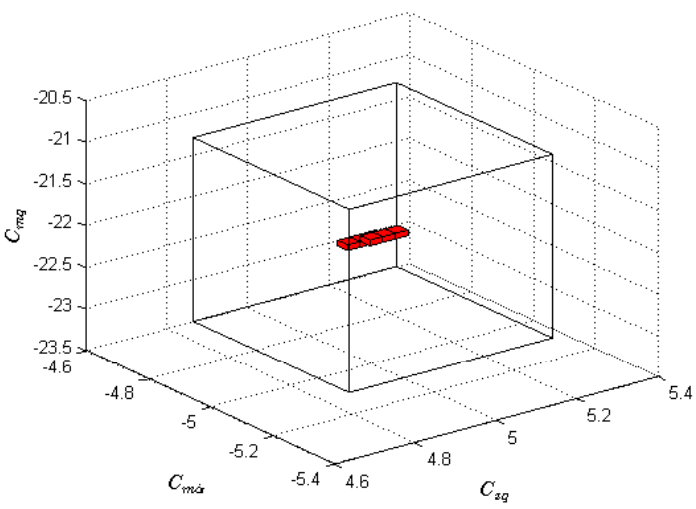

Figure 9: Acceptable sets $C_{z q}, C_{m \dot{\alpha}}, C_{m q}$ with optimal input.

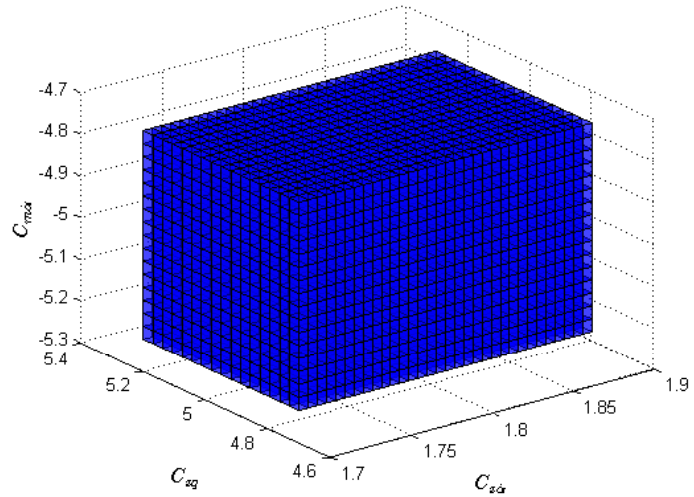

Figure 10: Rejected sets $C_{z \dot{\alpha}}, C_{z q}, C_{m \dot{\alpha}}$ with optimal input.

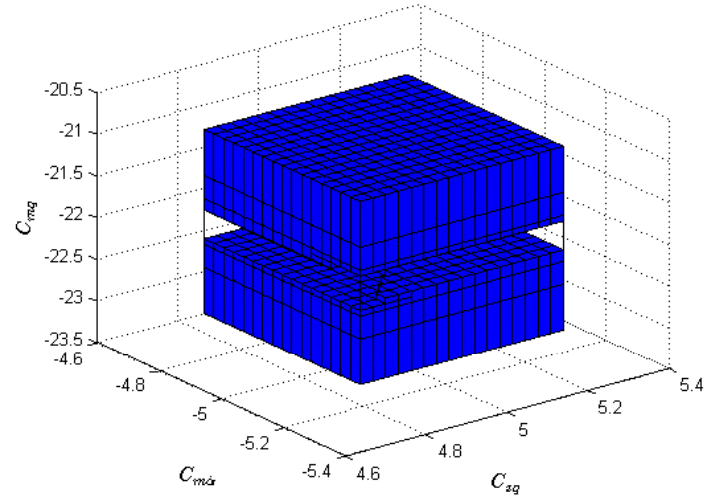

Figure 11: Rejected sets $C_{z \dot{\alpha}}, C_{z q}, C_{m \dot{\alpha}}$ with optimal input.

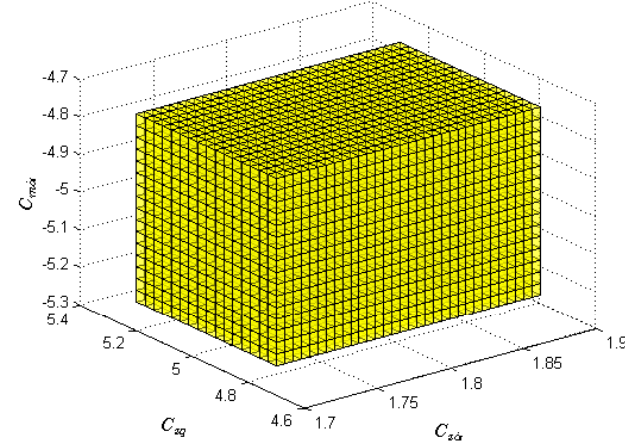

Figure 12: Undetermined sets $C_{z \dot{\alpha}}, C_{z q}, C_{m \dot{\alpha}}$ with optimal input.

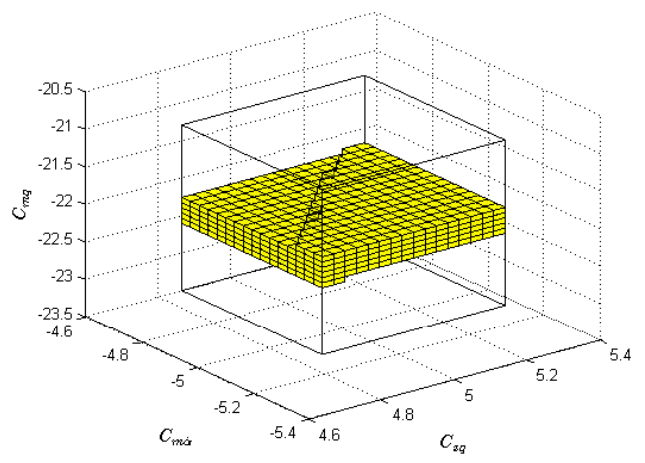

Figure 13: Undetermined sets $C_{z \dot{\alpha}}, C_{z q}, C_{m \dot{\alpha}}$ with optimal input.

We compare the results from the two inputs. The term $\% p$ indicates the percentage of unacceptable and uncertain interval sets we eliminated. Results have been done with constant input and optimal input. Clearly, the optimal input improves significantly the estimated parameters' domain. The last 3 rows of Table 1 show an improvement in estimation results. 
Table 1: Eliminated percentage of initial box.

\begin{tabular}{|c|c|c|}
\hline Parameter & $\% p_{\text {constant }}$ & $\% p_{\text {optimal }}$ \\
\hline$C_{z \dot{\alpha}}$ & 0.00 & 0.00 \\
\hline$C_{z q}$ & 0.00 & 75.00 \\
\hline$C_{m \dot{\alpha}}$ & 25.00 & 87.50 \\
\hline$C_{m q}$ & 65.62 & 93.75 \\
\hline
\end{tabular}

The volume of obtained acceptable boxes are presented in the following table:

Table 2: Volume of obtained acceptable boxes.

\begin{tabular}{|c|c|c|}
\hline Parameters & Constant input & Optimal input \\
\hline$C_{z \dot{\alpha}}, C_{z q}$ and $C_{m \dot{\alpha}}$ & 0.1215 & $9.4482 \mathrm{e}-04$ \\
$C_{z q}, C_{m \dot{\alpha}}$ and $C_{m q}$ & 1.7325 & 0.0116. \\
\hline
\end{tabular}

Through Table 2, we show the clear improvement of the acceptable domain for the parameters by using an optimal input. The first one (for parameters $C_{z \dot{\alpha}}, C_{z q}, C_{m \dot{\alpha}}$ ) and the second one (for parameters $C_{z q}$, $\left.C_{m \dot{\alpha}}, C_{m q}\right)$ are divided by 100 .

\section{CONCLUSION}

In this contribution, a procedure for parameter and state estimation in a bounded-error context has been pointed out. Two different inputs have been implemented and the estimation results have been compared. We can see that the coefficient $C_{z \dot{\alpha}}$ is difficult to be correctly estimated. The efficiency of the proposed algorithm combined with an optimized input has been pointed out. The presented method has potential for being used for active diagnosis problems in continuous-time systems or hybrid systems.

Our future works concern an improvement in the estimation parameter problem for these models and the potential application of this method to the active diagnosis. In fact, this last objective will be to use these tools to achieve an active diagnostic methodology that is to find a sequence of actions to refine the diagnosis.

As seen in the results for parameter estimation, the obtained results are clearly closed to the choice of input, thus another direction of our future work concerns the development of a methodology of optimal input design in a bounded error context for parameter estimation which is a new perspective.

\section{REFERENCES}

Belforte, G. and Gay, P. (2004). Optimal worst case estimation for lpv-fir models with bounded errors. Systems and Control Letters, 53:259-268.
Berz, M. and Makino, K. (1998). Verified integration of odes and flows using differential algebraic methods on high-order taylor models. Reliable Computing, 4:361 -369 .

Deville, Y., Janssen, M., and Hentenryck, P. (2002). Consistency techniques in ordinary differential equations. Constraints, 7:289-315.

Jauberthie, C. and Chanthery, E. (2013). Optimal input design for a nonlinear dynamical uncertain aerospace system. In IFAC Symposium on Nonlinear Control Systems, pages 469 - 474, Toulouse, France. IFAC.

Jauberthie, C., Verdière, N., and Travé-Massuyès, L. (2013). Fault detection and identification relying on set-membership identifiability. Annual Reviews in Control, 37:129-136.

Jaulin, L. (2009). A nonlinear set membership approach for the localization and map building of underwater robots. IEEE Transactions on Robotics, 25(1):88-98.

Jaulin, L., Kieffer, M., Didrit, O., and Walter, E. (2001). Applied interval analysis with examples in parameter and state estimation. Springer, London, 1nd edition.

Jaulin, L. and Walter, E. (1993). Set inversion via interval analysis for nonlinear bounded-error estimation. $A u$ tomatica, 29:1053 - 1064.

Kiefer, J. (1974). General equivalence theory for optimum designs (approximate theory). Annals of stat., 2:849 879.

Kieffer, M., Jaulin, L., and Walter, E. (2002). Guaranteed recursive nonlinear state bounding using interval analysis. International Journal of Adaptative Control and Signal Processing, 6:191 - 218.

Lohner, R. (1987). Enclosing the solutions of ordinary initial and boundary value problems. In Kaucher, E., Kulisch, U., and Ullrich, C., editors, Computer Arithmetic: Scientific Computation and Programming Languages, pages 255 - 286, Stuttgart. Wiley-Teubner.

Mehra, R. (1974). Optimal input signals for parameter estimation in dynamic systems - survey and new results. IEEE Vol.AC-19.

Moore, R. (1966). Interval analysis. Prentice Hall, New Jersey, 1nd edition.

Morelli, E. (1999). Flight test of optimal inputs and comparison with conventional inputs. Journal of aircraft, $36: 389-397$.

Nedialkov, N. (2006). Vnode-lp a validated solver for initial value problems in ordinary differential equations. Technical report, Dept. of Computing and Software.

Nedialkov, N., Jackson, K., and Pryce, J. (2001). An effective high-order interval method for validating existence and uniqueness of the solution of an ivp for an ode. Reliable Computing, 7:449 - 465.

Nedialkov, N. and Jackson, K. R. (2001). A new perspective on the wrapping effect in interval methods for initial value problems for ordinary differential equations. In Perspectives on Enclosure Methods, Vienna, Austria. Springer-Verlag.

Pasca, I. (2010). Formally Verified Conditions for Regularity of Interval Matrices. In Lecture notes in artificial intelligence, volume 6167. Springer. 
Pronzato, L. and Walter, E. (1988). Robust experiment design via maximin optimization. Mathematical Biosciences, 89:161 - 176 .

Rauh, A. and Auer, E. (2011). Modeling, design and simulation of systems with uncertaintites. Springer, Berlin, 1nd edition.

Rihm, R. (1994). Interval methods for initial value problems in odes. In IMACS-GAMM International Workshop on Validated Computations, Amsterdam. Elsevier.

Rojas, C., Welsh, J., Goodwin, G., and Feuer, A. (2007). Robust optimal experiment design for system identification. Automatica, 43:993 - 1008.

Walter, E. and Pronzato, L. (1994). Identification de modèles paramétriques à partir de données expérimentales. Masson. 